\title{
A Recurrent Multimodal Network for Binding Written Words and Sensory-Based Semantics into Concepts
}

\author{
Andrew P. Papliński and William M. Mount \\ Monash University, Australia
}

\author{
Lennart Gustafsson \\ Luleå University of Technology, Sweden
}

\begin{abstract}
We present a recurrent multimodal model of binding written words to mental objects or concepts and investigate the capability of the network in reading misspelt but categorically related words. Our model consists of three mutually interconnected association modules which store mental objects, represent their written names and bind these together to form mental concepts. A controllable feedback gain term controlling top-down influence is incorporated into the model architecture and it is shown that correct settings for this during map formation and simulated reading experiments is necessary for correct interpretation and semantic binding of the written words.
\end{abstract}

\section{Introduction}

We use a network of recurrent self-organizing modules to model aspects of the reading process within the cortex. As perceptions of the world around us are experienced by combining sensory inputs of different modalities with internal world models learned within the mind, our network consists of models of five cortical areas, two of which process the sensory information and three others to represent a twolevel hierarchical model of the world. Such an architecture is motivated by the fact that the neural processing first takes place in mainly unimodal (visual, auditory, etc.) hierarchies in the brainstem and sensory cortices of the cerebrum. The unimodal percepts then converge in multimodal association areas such as STS (Superior Temporal Cortex). At this level we have highly abstracted, semantic representations of objects. We attach words to these mental objects and thus build conceptual representations.

The world in our work consists of a set of animals defined in terms of perceptual features and qualities and we bind the written names of these to the learned mental objects representing them. The processing and binding of written names to mental objects follows a similar methodology to a previous model used in lexical binding of spoken names (Papliński, Gustafsson, and Mount 2010). However, we have improved working of the network by adding feedback gains to control the level of modulation feedback from the modeled bi-modal integration area.

Copyright (C) 2011, Association for the Advancement of Artificial Intelligence (www.aaai.org). All rights reserved.
At a general level we are concerned with aspects of processing and binding of multimodal information and are influenced in this by works such as (Calvert, Campbell, and Brammer 2000; Rolls 2004; Mesulam 1998). In particular, it is known that tasks involving identification of animals as a category apart from tools, for example, causes activity in parts of left inferotemporal (IT) cortex (Tranel et al. 2005), whereas the more demanding task of identifying different animal species causes activity also in anteromedial temporal cortex, an area employed in more fine-grained discrimination between objects (Bright, Moss, and Tyler 2004; Bright et al. 2007).

The neuronal circuitry involved in reading is undoubtedly complex and much current research concentrates on an area called VWFA (Visual Word Form Area) in left fusiform gyrus (McCandliss, Cohen, and Dehaene 2003)) where prelexical, i.e., strings of letters, and lexical processing of word forms (Glezer, Jiang, and Riesenhuber 2009) takes place. One of the more complete representations of cortical areas involved in the process of reading resulting from intensive fMRI investigation is given in (Dehaene 2009). Chapter 2, Fig. 2 (available electronically) presents 13 cortical mutually interconnected areas involved in a process of reading arranged in the five groups, namely: visual input, visual word form, access to meaning, access to pronunciation and articulation and top-down attention and serial reading.

In our work we use a much simplified model consisting of just five 'cortical' areas. Due to the fact that the resulting recurrent network exhibits non-linear dynamics that can be difficult to predict, it is encouraging to note that the model presents a range of interesting and psychophysically plausible results. One of the fundamental concepts employed in our modelling framework is the concept of a ubiquitous "neuronal code", which implies a unified way of representing information exchanged by modules of the network.

At the beginning of our consideration is the problem of coding words in neocortex, since several methods based around letter combinations and positioning with increasing sophistication have been proposed (Davis and Bowers 2006; Grainger 2007; Whitney 2008). We will employ here a relatively straightforward word coding method called open bigram coding as presented in (Whitney 2008; Dehaene 2009) and described further in Section 3.1. 
The other fundamental consideration is how and where conceptual representations are stored in neocortex. This is an active field of research with competing hypotheses; either there are separate representations for words and for sensory representations, or else all representations converge onto a unitary system of representations common for both words and sensory representations. A 'compromise' between these hypothesis is possible as presented in (Bright, Moss, and Tyler 2004). Accordingly, we build our model on the unitary system as argued there.

\section{Model Description}

Our model belongs to the class of models using maps that describe mapping multidimensional sensory signals into a low-dimensionality cortical representation. We direct the reader's attention to (Schreiner and Winer 2007) for a discussion on the existence and significance of cortical maps.

As a hierarchical model of reading combining bottomup sensory integration with top-down attentional processing, our model follows principles similar to those presented in (Graboi and Lisman 2003), which describes a multilayered model for processing of features, letters and words in cortex and which can account for several key findings from human word search, semantic priming and attention shifting experiments. For a related neurocomputational account of map-based processing and its role in language and speech comprehension and production, see also (Li, Zhao, and MacWhinney 2007; Mayor and Plunkett 2010).

In our approach, which flows from our earlier works on multi-modal integration ((Papliński, Gustafsson, and Mount 2010) and predecessors cited there), self-organized modules form low dimensional labels. ${ }^{1}$ These labels are used as afferent signals to up-stream modules, and may represent any type of perceptual, conceptual or lexical 'features'. Such universal feature labelling is consistent with the neurocomputational modelling approach of (Gliozzi et al. 2009).

As noted above, we do not attempt to represent each and every cortical area taking part in perceptual and semantic processing of mental objects or in higher-level language and related cognitive tasks. Rather our aim is to represent a subset of language processing in a smaller number of modules aggregating the processes of several cortical areas. The model consists of 5 processing modules divided into 3 layers, connected as shown in Figure 1.

The processing pathway for visual and associated perceptual information for the mental object is depicted on left hand side of the figure ( $\mathrm{P}$ and $\mathrm{MO}$ ) while word processing paths are included on the right(Wrd and UW). These pathways then converge and binding occurs within a hypothetical high-level bimodal association area, M+W. In this sense the model is consistent with that outlined by (Caramazza et al. 1990) which claims that all processing routes involved

\footnotetext{
${ }^{1}$ In general, maps and their interconnections are trained using the Kohonen learning law (Kohonen 2001), normalised Hebbian learning, or combination of both as in (Miikkulainen 1997; Sit and Miikkulainen 2009; Miikkulainen and Kiran 2009; Monner and Reggia 2009; Li, Zhao, and MacWhinney 2007; Mayor and Plunkett 2010).
}

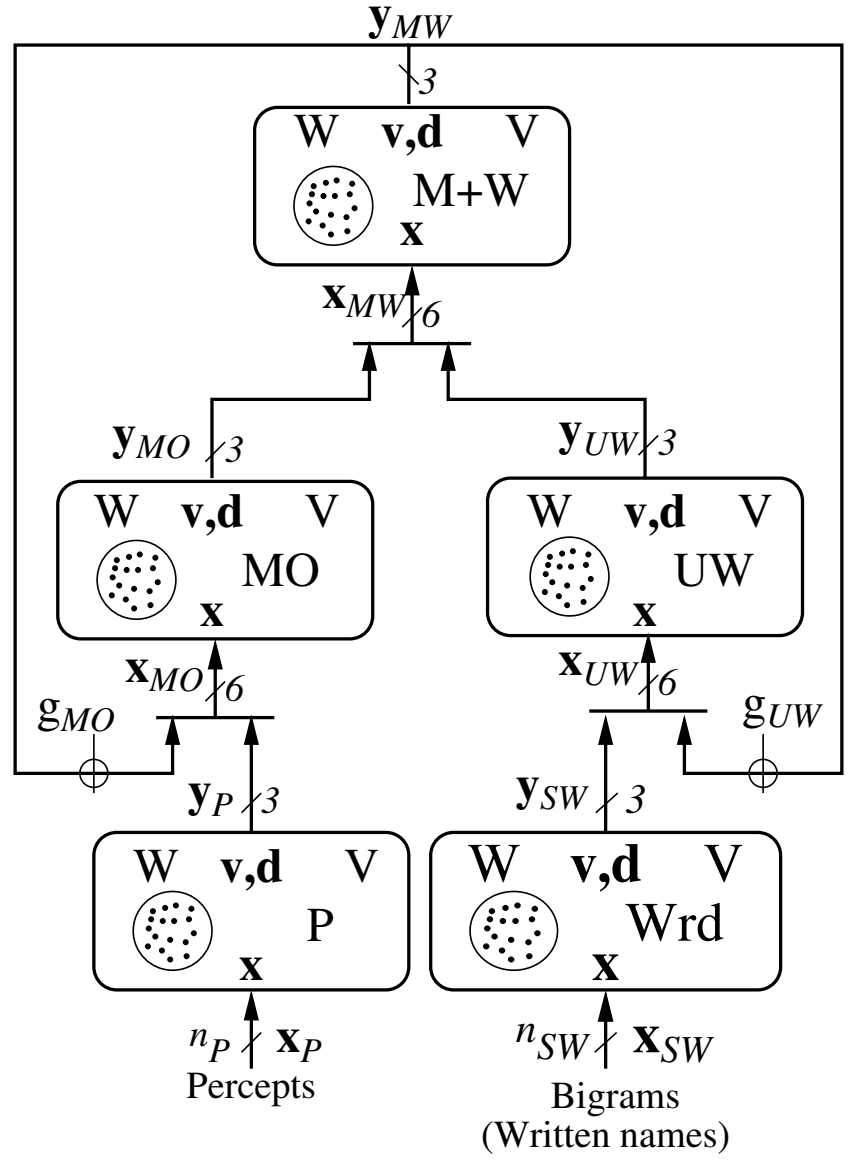

Figure 1: The network of interconnection simulated cortical maps: Wrd - Sensory Word map, UW - Unimodal Association Word map, $\mathrm{P}$ - Sensory Percepts map, MO - Mental Objects map, $\mathrm{M}+\mathrm{W}-$ Bimodal Association map: mental objects and written names

tasks such as reading converge on a single set of conceptual representations common to both modalities.

Each module consists of a number of artificial neuronal units randomly located in a circular area. Relative positions of 'neurons' inside the circle are described by a position matrix $\mathbf{V}$. The total number of neurons is selected in proportion to the number of objects represented by the module, with rows in the weight matrices $\mathbf{W}$ characterizing the synaptic strengths in each module. The main functions for each map is described below:

- Percepts map (P) encodes basic perceptual features, such as size, colour, form of locomotion and social behaviour for each object within the category: Animals

- Mental Objects map (MO) is a map of perceivable mental objects and semantic relationships for the object category, i.e. a topographically organised map of a set of 30 animals according arranged by perceptual features.

- Word map (Wrd) models letter and letter position processing in VWFA. Input to this module take the form of 
word bi-grams based on the possible pairings of $26 \mathrm{En}$ glish/Latin letters.

- Unimodal Word map (UW) performs sub-lexical processing of words and projects these as written names for lexical binding within the bi-modal association map.

- 'Bimodal' association map $(\mathrm{M}+\mathrm{W})$ is responsible for the lexical binding of perceivable mental objects and sensory based semantics to form labelled mental concepts.

Conceptually there are two types of modules, sensory modules and association modules, reflecting the layered architecture in Figure 1. Sensory modules operate on a relatively large number of afferent signals and produce a lowdimensionality efferent signal labeling the sensory object. This label information consists of a three-dimensional vector encoding the relative location of the most excited neuron within a given cortical patch, supplemented by the postsynaptic activity level of this neuron. Such labels form a universal representation of all information passed between object maps within the hierarchical model. Association modules, in turn use these labels as their afferent signals and produce efferent labels encoding positions of activated neuronal patches within these maps. The operation of a cortical module is functionally equivalent to mapping the higher dimensionality input space to a three-dimensional output space this a fundamental property of our model.

\section{Operation of Recurrent Network}

The processing modules within the network model are interconnected via feedforward sensory processing pathways and feedback connections from the bimodal integration module $(\mathrm{M}+\mathrm{W})$, as indicated in Figure 1. Feedback gain terms, $g_{m o}$ and $g_{u w}$ are also shown on these recurrent pathways. The effect of these is discussed below and in Section 3.2.

It is assumed that during normal operation association modules perform static non-linear mappings of the form:

$$
\begin{aligned}
\mathbf{y}_{\mathbf{M W}} & =f\left(\mathbf{W}_{\mathbf{M W}} \cdot \mathbf{x}_{\mathbf{M W}}\right), \mathbf{x}_{\mathbf{M W}}=\left[\mathbf{y}_{\mathbf{M O}}, \mathbf{y}_{\mathbf{U W}}\right] \\
\mathbf{y}_{\mathbf{M O}} & =f\left(g_{M O} \cdot \mathbf{W}_{\mathbf{M O}_{\mathbf{M W}}} \cdot \mathbf{y}_{\mathbf{M W}}+\mathbf{W}_{\mathbf{M O}} \cdot \mathbf{y}_{\mathbf{P}}\right) \\
\mathbf{y}_{\mathbf{U W}} & =f\left(g_{U W} \cdot \mathbf{W}_{\mathbf{U W}_{\mathbf{U W}}} \cdot \mathbf{y}_{\mathbf{M W}}+\mathbf{W}_{\mathbf{U}} \mathbf{W}_{\mathbf{S W}} \cdot \mathbf{y}_{\mathbf{S W}}\right)
\end{aligned}
$$

where $\mathbf{x}$ and $\mathbf{y}$ represent input and output signals, respectively and $f(\cdot)$ describes the Winner-Takes-All function.

Adding feedback pathways to a highly non-linear multilayered network presents a challenge as it results in a complex, dynamical system with a rich set of behaviors which may become unpredictable and chaotic. For the bi-modal association module we can write the following formal dynamic equation:

$$
\mathbf{y}_{\mathbf{M W}}(t+1)=\mathcal{M}\left(\mathbf{y}_{\mathbf{M W}}(t), \mathbf{y}_{\mathbf{M O}}(t), \mathbf{y}_{\mathbf{U W}}(t)\right)
$$

the purpose of this, however, is mainly to emphasize the recurrent and non-linear nature of the network. Nonetheless, from the simulation perspective, the trained network is observed to settle immediately if we apply exogenous stimuli that are congruent with the endogenous thoughts or initial conditions. In other words, if the labels in the network are known and congruent, the network quickly converges to a stable state. See (Papliński, Gustafsson, and Mount 2010) for behaviour of a similar network for incongruent inputs.

A brief description of the preprocessing, training and operation of the network is as follows:

\subsection{Preprocessing of percepts and word bi-grams}

Prior to training of the maps, a simple preprocessing step is performed to produce the perceptual based semantic and letter bi-gram information for the separate perceptual and written word/lexical pathways. Open bi-gram encoding (add ref) is used in the present model. The purpose of this is to encode the attribute lists of the former and relative letter positioning of associated words for the later into a consistent numerical format for the self-organised maps. To ensure computational efficiency all inputs and weight vectors are projected on the unity hypersphere. Working with unity vectors makes it easy to compare them by calculating relevant inner products and allows us to use a simplified dotproduct learning law (Kohonen 2001).

\subsection{Sequential development of maps}

The map training sequence approximately follows that of the widely accepted model of neural ontogenesis and cortical map formation (add ref). In particular, the processing and adaptation to sensory and learned label information is propagated in a feedforward or bottom-up direction and feedback processing subsequently comes into play in a recurrent optimisation of the higher level maps. ${ }^{2}$

Feedforward training of unimodal sensory maps The first training step involves initial organisation of the unimodal maps, MO and UW in Figure 1. To train the map of mental objects, $\mathrm{MO}$, feature vectors $\mathbf{x}_{\mathbf{P}}$ describing the animals are first encoded by the auxiliary module, $\mathrm{P}$ as a dimensionally reduced label, $\mathbf{y}_{\mathbf{P}}$ and used as input to MO. Competitive learning process then encodes these inputs as a map of Mental Objects organised according to their perceptual semantics. Following training, the object categorizing module $\mathrm{P}$ is disconnected and the signal $\mathrm{y}_{\mathbf{P}}$ is interpreted as thought commands used for recalling mental objects stored with the module MO. The UW map is trained independently using the topographically organised word bi-gram representations from the Wrd module.

Feedforward training of bimodal integration map The next step is to train the bi-modal map $\mathrm{M}+\mathrm{W}$ using combined inputs from $\mathrm{MO}$ and UW. Through statistical pairing of randomly presented mental object and word label information from each of the unimodal maps, an initial bimodal map of lexically-bound concepts is formed, in this case of a named set of animals.

Feedback training of sensory and bimodal maps Following completion of the feedforward training steps, the three association modules forming the recurrent part of the

\footnotetext{
${ }^{2}$ Note that while no recurrent feedback from higher level modules is used in the initial feedforward training steps, selforganisation of the maps assumes local recurrent connections across the map output layer in order to implement the required the competitive learning process.
} 
network are trained together. This time, after each learning step we perform several relaxation steps running the network as in eqn (??), until all efferent signals in the network are constant. There is a limit on the number of iterations imposed that is important in the initial learning stages. Once the maps are fully developed, the network stabilizes quickly after a small number of iterations depending on congruence between the perceived mental objects and associated written words or names.

As a result of this training step, the unimodal maps, MO and UW are optimised and re-organised to reflect contextual information transmitted from the bimodal integration layer. The $\mathrm{M}+\mathrm{W}$ module in turn is adjusted so as to represent the statistical correlations between in the unimodal data encoded in the label information $\mathbf{y}_{\mathbf{m o}}$ and $\mathbf{y}_{\mathbf{u w}}$. An important feature of the model architecture presented in Figure 1 are the feedback gain terms, $\mathbf{g}_{\mathbf{m o}}$ and $\mathbf{g}_{\mathbf{u w}}$. The implementation of this variable feedback is a new addition to previous models presented in (Papliński, Gustafsson, and Mount 2010) and predecessors cited there. In order to ensure that inputs to the MO and UW modules are properly bounded and that weight vectors remain on the unity hypersphere, a novel algorithm is employed in which the inputs and gain values are effectively spatially decomposed and then reassembled within the cortical neurons. (check this)

\subsection{Testing response of network to word stimuli}

Following training of the maps, the operation of the network is essentially as follows: The written word excites cortical patches in the word bi-gram map, Wrd, and via the forward path from this low-level sensory area, patches within the unimodal association area UA and bimodal binding area, $\mathrm{M}+\mathrm{W}$ are also excited. A similar pattern of activation is produced via the feed-forward pathway from the 'perceptual' mental objects area MO to $\mathrm{M}+\mathrm{W}$. These direct feed-forward paths assure that the binding process is rapid - at least in the case of congruous thoughts and inputs. In the case of discrepancy or incongruity between the mental object and a written word (for example misspelt word, or mismatch between word and perceived object) a feedback loop is automatically activated from the $\mathrm{M}+\mathrm{W}$ area back to the to $\mathrm{MO}$ and UA areas. This initiates a recurrent cycle that typically converges on a globally sensible solution, assuming that no contradictory input (or "evidence") is presented and that one actually exists.

Another novel aspect in the present version of the selforganising multimodal framework is that the feedback gain terms $\mathbf{g}_{\mathbf{m o}}$ and $\mathbf{g}_{\mathbf{u w}}$ used in feedback training step described above can also be set separately during the testing or operational phase. In this way the effect of controllable feedback, and implicit "top-down mediated selective attention" upon the hierarchically-organised set of maps trained with varying degrees of feedback can be explored. An initial set of results showing some of these effects are presented below.

\section{Results}

Preliminary results show the development of activities over time in the modules of our simulated network when thought commands about different mental objects, in this case animals, and their associated written names are simultaneously presented. This perceptual or mental binding task is considered central to language understanding during the activity of reading. The simulation scenario is also similar to the fMRI-based study of recognition of spoken words representing animals when subjects are been cross-modally primed for different animals (Noppeney et al. 2008).

As an example of the operation of the network in recognition of words corresponding to known mental objects, consider the situation where the multimodal computational network is initialised to a particular animal, while being presented with a word more closely matching the name of another animal and which has a similar spelling. The response of the multimodal network model to this situation is presented in Figures 2 and 3.

In the maps depicted in these figures, neuronal positions are marked with the yellow dots and the map area is tessellated with respect to the peaks of neuronal activities for each stimulus. We can recognize the patch for various animals, e.g. 'dog', 'frog', 'goat' and compare it with the activity surface for the same stimulus in the figure (how to see activity surface?. The objects or written names are 'placed' in the relevant cortical area during the learning process. Once the learning is completed, the cortical area responds with an activity pattern characteristic to each stimulus.

At the starting point, the MO module thinks 'dog', whereas the unimodal word association module UW reads the orthographically similar word 'grog'. The bimodal association map, $\mathrm{M}+\mathrm{W}$ is arbitrarily initialized with a mental object 'dog'. This initial state is marked on three maps in Figures 2 and 3 with the ' $\diamond$ '. Since these positions have been learned during the training procedure, the relative postsynaptic strengths are initially at their maximum value, as seen in the upper right hand side of both figures.

In the first case, Figure 2, a relatively low feedback gain, $g_{u w}=g_{m o}=0.6$ has been used during the reading test phase. In this scenario, although the bimodal map $\mathrm{M}+\mathrm{W}$ and mental objects map, MO clearly converge to a 'frog' solution, the response of the unimodal word area UW arrives at a point on the class boundary between 'frog' and 'goat'. This suggests some uncertainty in recognition of the word for the animal most closely matching the word-stimulus ('grog'), implying that the network as a whole has not been able to successfully bind the correct name 'frog' to the corresponding mental object. This level of uncertainty is indicated by the final value of relative post-synaptic strengths, in which the response of $\mathrm{MO}$ and $\mathrm{M}+\mathrm{W}$ is stronger than that of $\mathrm{UW}$.

Now compare this with the situation in Figure 3 when a greater level of feedback gain, $g_{u w}=g_{m o}=0.9$ has been used during testing. In this case the network quickly settles to a solution when the thought prevails; the final response of all maps including UW is consistent and the network as whole converges rapidly on the 'frog' conclusion. This level of confidence in this outcome is further indicated by the high levels of the relative post-synaptic strengths.

Through this real-time correction of the misspelt distractor word 'grog' during a simulated reading experiment, this result demonstrates how "semantic priming" across a known set of objects within an mental object category can be effectively represented within the model. We hope that this sim- 

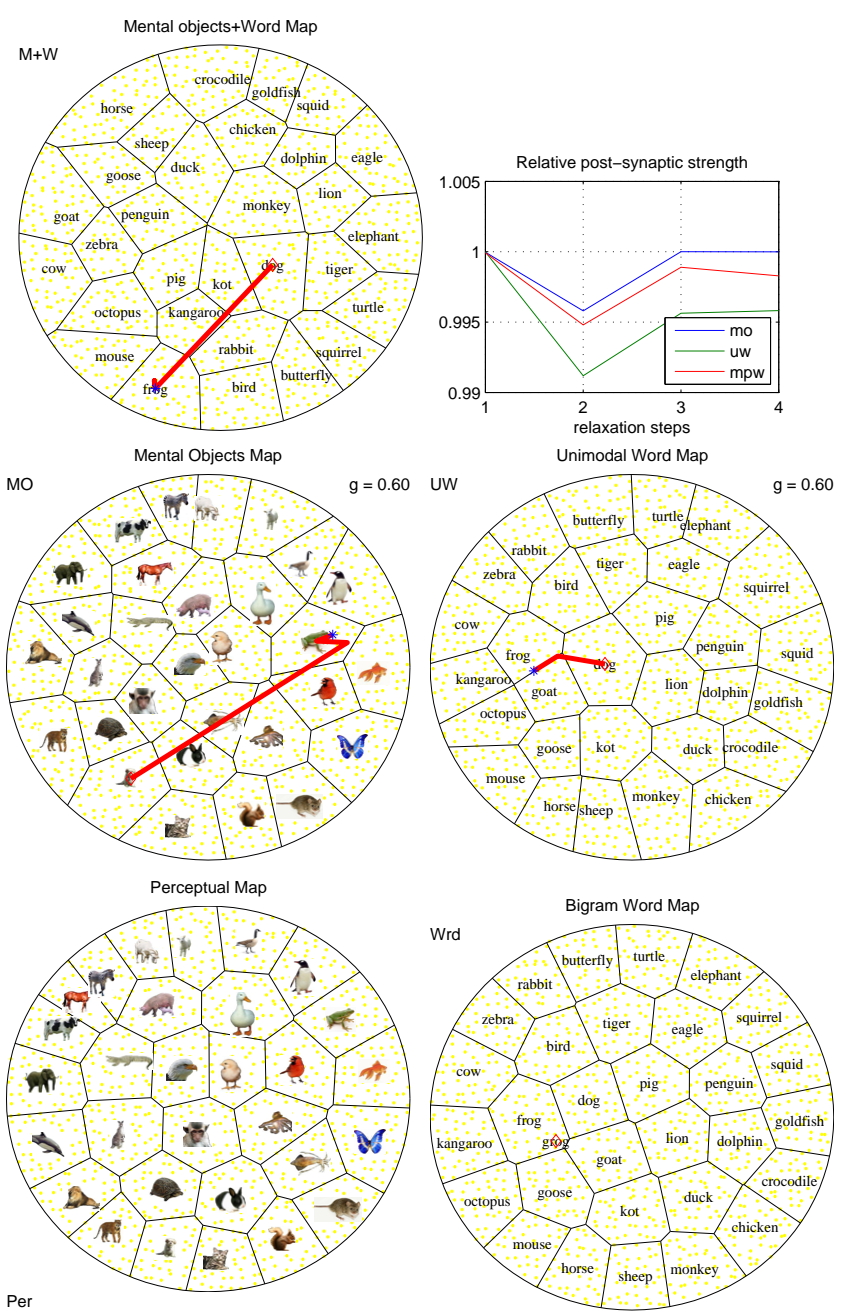

Figure 2: "frog-grog" - low feedback gain case $(g=0.6)$

ple example illustrates the power of presented computational model and its potential application in related investigations.

\section{Discussion}

For the a direct comparison to be possible, it is necessary that exactly the same trained hierarchical network model be used in each case. In this case, a feedback gain setting of $g_{u w}=g_{m o}=0.6$ was used during recurrent phase in the formation process (as described in Section 3.2). More varied simulation results can be obtained if different feedback gain values are used, however due to limited space available examples of the types of aberrant behaviours that can be produced as a result are not considered at this time.

In general, reducing the feedback gain during map formation will result in a overall network that responds very well to new and less predictable inputs (such as words and nonwords) but which lacks the contextual 'knowledge' required to correctly associate these inputs with crossmodal percepts and mental objects. Conversely, applying to great a level of feedback during recurrent training step results in a network
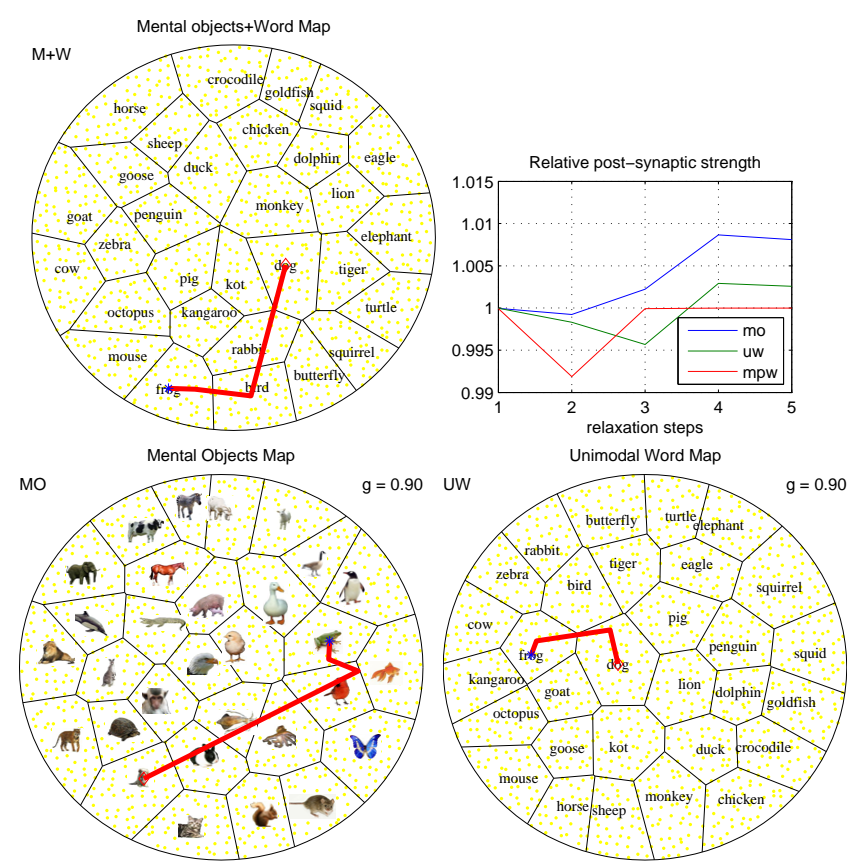

Figure 3: "frog-grog" - high feedback gain case $(g=0.9)$

with a tendency to become locked up in previously known states or thoughts and less able to adapt to new sensory information.

This suggests that an optimal level of feedback gain is required in order to realise an reading network which can effectively employ previously learned knowledge to correctly perceive and learn new words.

One possibility for future research would be to use the feedback gain within an incremental learning regime in which global reinforcement feedback is used to assess the 'utility' of the learned set of maps at a particular setting of feedback (initial condition) versus feedforward (input discrepancy) bias. The feedback gain $g$ could then be decreased if the network became 'stuck' and unable to adapt well to new conditions and increased if a stronger belief in prior conditions was deemed to result in a better overall performance. Adopting of such a 'self-supervised' approach could be a way to incorporate a process analogous to consciously controlled selective attention in a straightforward and integrated way which works to optimise efficiency of the learning process.

\section{Conclusion}

We have presented a model for binding written names to perceptually-based semantic objects and provide preliminary results to demonstrate how this can be used in modelling cognitive functions basic to reading. This includes automatic 'correction' of misspelt words when a similar known word that is bound to an active mental object or by extension, object category is attended to. Such cognitive processes are of fundamental importance to particular human activity of reading. By introduction of controllable feed- 
back gain the behavioural repertoire of the model is greatly increased presenting an opportunity to explore a number of other effects on learning and cognitive behaviour within the outlined computational framework.

In the interest of maintaining structural simplicity, several assumptions were made in the model. For convenience specific visual and other perceptual modalities, conceptual categories and semantic relationships are combined in a 'mental objects map'. This simplification is computationally efficient as it allows conceptual information to be encoded as arbitrary lists of features and object qualities. In a more comprehensive and biologically realistic model, the auxiliary ' $\mathrm{P}$ ' module could be divided into specific sensory modalities or sub-modalities, used to represent visual, tactile, spatial or other modal features relevant to mental object categories such as plants, animals or tools.

The representation of the mental objects would then involve a multi-modal integration of such features and lexical binding of these to their associated written or spoken names. From the lessons gained through this modelling exercise as well that gained through experiments in lexical binding of spoken names to mental objects, we hope to extend the model to integrate structurally separate processing pathways and perform multimodal and possibly transmodal binding across auditory and written lexical and perceptually grounded modalities.

\section{References}

Bright, P.; Moss, H. E.; Longe, O.; Stamatakis, E. A.; and Tyler, L. K. 2007. Conceptual structure modulates anteromedial temporal involvement in processing verbally presented object properties. Cerebral Cortex 17:1066-1073.

Bright, P.; Moss, H. E.; and Tyler, L. K. 2004. Unitary vs multiple semantics: Pet studies of word and picture processing. Cerebral Cortex 89:417-432.

Calvert, G.; Campbell, R.; and Brammer, M. 2000. Evidence from functional magnetic resonance imaging of crossmodal binding in the human heteromodal cortex. Current Biology 10:649-657.

Caramazza, A.; Hillis, A. E.; Rapp, B. C.; and Romani, C. 1990. The multiple semantics hypothesis: Multiple confusions? Cogn. Neuropsych. 7:161-189.

Davis, C. J., and Bowers, J. S. 2006. Contrasting five different theories of letter position coding: Evidence from orthographic similarity effects. J. Exp. Psych.: Human Perception and Performance 32(3):535-557.

Dehaene, S. 2009. Reading in the Brain. Viking. http:// pagesperso-orange.fr/readinginthebrain/figures.htm.

Glezer, L. S.; Jiang, X.; and Riesenhuber, M. 2009. Evidence for highly selective neuronal tuning to whole words in the "visual word form area". Neuron 62(2):199-204.

Gliozzi, V.; Mayor, J.; Hu, J.-F.; and Plunkett, K. 2009. Labels as features (not names) for infant categorisation: A neuro-computational approach. Cog. Sci. 33(3):709-738.

Graboi, D., and Lisman, J. 2003. Recognition by top-down and bottom-up processing in cortex: The control of selective attention. J. Neurophysiol. 90:798-810.
Grainger, J. 2007. Cracking the orthographic code: An introduction. Language and Cognitive Processes 23(1):135 .

Kohonen, T. 2001. Self-Organising Maps. Berlin: SpringerVerlag, 3rd edition.

Li, P.; Zhao, X.; and MacWhinney, B. 2007. Dynamic selforganization and early lexical development in children. $\mathrm{Neu}$ ron 31:581-612.

Mayor, J., and Plunkett, K. 2010. A neurocomputational account of taxonomic responding and fast mapping in early word learning. Psychol Rev. 117(1):1-31.

McCandliss, B. D.; Cohen, L.; and Dehaene, S. 2003. The visual word form area: expertise for reading in the fusiform gyrus. TRENDS Cog. Sci. 7(7):293-299.

Mesulam, M.-M. 1998. From sensation to cognition. Brain 121:1013-1052.

Miikkulainen, R., and Kiran, S. 2009. Modeling the bilingual lexicon of an individual subject. In Lect. Notes in Comp. Sci., volume 5629, 191-199. Springer.

Miikkulainen, R. 1997. Dyslexic and category-specific aphasic impairments in a self-organizing feature map model of the lexicon. Brain and Language 334-366.

Monner, D., and Reggia, J. A. 2009. An unsupervised learning method for representing simple sentences. In Proc. Int. Joint Conf. Neural Net., 2133-2140.

Noppeney, U.; Josephs, O.; Hocking, J.; Price, C.; and Friston, K. 2008. The effect of prior visual information on recognition of speech and sounds. Cerebral Cortex 18:598609.

Papliński, A. P.; Gustafsson, L.; and Mount, W. M. 2010. A model of binding concepts to spoken names. Australian Journal of Intelligent Information Processing Systems 11(2):1-5.

Rolls, E. T. 2004. Multisensory neuronal convergence of taste, somatosentory, visual, and auditory inputs. In Calvert, G.; Spencer, C.; and Stein, B. E., eds., The Handbook of multisensory processes. MIT Press. 311-331.

Schreiner, C. E., and Winer, J. A. 2007. Auditory cortex mapmaking: Principles, projections, and plasticity. Neuron 56(2):356-365.

Sit, Y. F., and Miikkulainen, R. 2009. Computational predictions on the receptive fields and organization of V2 for shape processing. Neural Computation 21(3):762-785.

Tranel, D.; Grabowski, T. J.; Lyon, J.; and Damasio, H. 2005. Naming the same entities from visual or from auditory stimulation engages similar regions of left inferotemporal cortices. J. Cogn. Neurosci. 17(8):1293-1305.

Whitney, C. 2008. Comparison of the seriol and solar theories of letter-position encoding. Brain and Language 107:170-178. 